Bonetti, D. 45

Clarkson, J.E. 45

Csikar, J.I. 50

Dasanayake, A.P. 68

Douglas, G.V.A. 50

Ekstrand, K.R. V, 15

Fontana, M. 22
Hill, R. 68

Hirsch, S. 68

Keller, M.K. 38

Lamster, I.B. 78

Mascarenhas, A.K. 1

Neidell, M. 78

Pretty, I.A. V, 9
Ramsdale, M.P. 50

Shearer, B. 78

Tellez, M. V, 61

Twetman, S. 38

Vinall-Collier, K. 50

Wilson-Genderson, M. 68

Wolff, M.S. 61, 68

\title{
Subject Index Vol. 50, Suppl. 1, 2016
}

1,000 ppm 1

Adolescents 9

Amorphous calcium phosphate 22

Arginine 22

\section{Calcium 22}

Caries 9, 38

- prevalence 1

- severity 1

Casein phosphopeptide 22

Children 9

Clinical trials 22

Cost-effectiveness 78

Dental caries 45

- guidelines 50

- sealants 78
Effectiveness 9, 15

Epidemiology 1

Evidence-based dentistry 45

- practice 45

Fluoride 1, 22, 38, 61

- varnish 45, 50,78

- $\quad$ as public health intervention 68

High fluoride concentration toothpaste 50

- - dentifrices 9,15

High-risk populations 1

Knowledge translation 45

Oral health 45

- - workforce diversification 68
Polyphenol 22

Populations 1

Prevention 38

Public health 61

- - intervention 68

Remineralization 22

Root caries 38

- - lesion 15

Schoolchildren 38

Toothpaste 61

Varnish 61

Xylitol 22 